\title{
The BCS model and the off shell Bethe ansatz for vertex models
}

\author{
Luigi Amico ${ }^{[1]}$, G. Falci ${ }^{[1,2]}$, and Rosario Fazio ${ }^{[1]}$ \\ [1] Dipartimento di Metodologie Fisiche e Chimiche (DMFCI), Universitá di \\ Catania, viale A. Doria 6, I-95125 Catania, Italy \\ Istituto Nazionale per la Fisica della Materia, Unitá di Catania, Italy \\ [2] Laboratoire d'Etudes des Propriétés Electroniques des Solides, Centre \\ National de la Recherche Scientifique, BP 166, 38042 Grenoble Cedex 9, \\ Grenoble, France
}

\begin{abstract}
We study the connection between the BCS pairing model and the inhomogeneous vertex model. The two spectral problems coincide in the quasiclassical limit of the off-shell Bethe Ansatz of the disordered six vertex model. The latter problem is transformed into an auxiliary spectral problem which corresponds to the diagonalization of the integrals of motion of the BCS model. A generating functional whose quasi classical expansion leads to the constants of motion of the BCS model and in particular the Hamiltonian, is identified.
\end{abstract}

PACS: 03.65.Fd, 74.20.Fg

keywords: Integrable models; BCS Superconductivity.

\section{Introduction}

One of the most successful models of interacting electrons is the BCS model of pairing [1]. Originally proposed to describe properties of superconductors [2], the pairing idea has been applied to a large variety of physical systems in nuclear physics [3] and in QCD [4. Recent experiments in metallic nanoparticles $[5$ have renewed the interest in the problem of pairing correlations in mesoscopic systems [6]. The BCS Hamiltonian consists of a kinetic and an interaction term which describes the attraction between electrons in time reversed states

$$
H=\sum_{\substack{j=1 \\ \sigma=\uparrow, \downarrow}}^{\Omega} \varepsilon_{j \sigma} c_{j \sigma}^{\dagger} c_{j \sigma}-g \sum_{j, j^{\prime}=1}^{\Omega} c_{j \uparrow}^{\dagger} c_{j \downarrow}^{\dagger} c_{j^{\prime} \downarrow} c_{j^{\prime} \uparrow} \quad .
$$

The quantum number $j \in\{1, \ldots, \Omega\}, \sigma \in\{\uparrow, \downarrow\}$ labels a shell of doubly degenerate time reversed states of energy $\epsilon_{j} ; c_{j, \sigma}$ and $c_{j, \sigma}^{\dagger}$ are the corresponding electronic operators; $g$ is the BCS coupling constant. The low energy properties associated to this model are universal functions of the ratio between two energies, the single particle average level spacing and the BCS gap $[6]$.

Various exact results have been obtained for the BCS Hamiltonian.

In the limit $g \rightarrow \infty$ the exact eigenvalues and eigenstates can be found (see for example Ref. [7]) and the integrals of motion are Gaudin Hamiltonians [8]. An important consequence of the relation with the isotropic Gaudin magnet (discussed in Appendix A), is that the Quantum Inverse Scattering Method (QISM) [9] for the $g \rightarrow \infty$ BCS 
model can be related with the QISM for the Gaudin model [11]. The $g \rightarrow \infty$ BCS model can be also related to the inhomogeneous vertex models [12, 13].

Much less work has been done for finite $g$. The exact solution was found by Richardson and Sherman (RS) [19] and independently by Gaudin [20] by means of the Bethe Ansatz (BA) technique. Approximate expression of correlation functions were found in the Ref. [21]. More recently, the integrals of motion of the BCS model were obtained [22, 11] and were diagonalized by means of the algebraic BA.

In this work we show that for finite $g$ the BCS model is connected to a disordered six vertex model through the Off-Shell BA (OSBA) introduced by Babujian et al. in Refs. [17, 18]. In this framework, the known connection between the isotropic Gaudin models and the inhomogeneous vertex model is obtained as the Mass-Shell limit which corresponds to $g \rightarrow \infty$.

A strong hint towards our result is provided by a recent work by Sierra [23] who has shown connection between the BCS pairing model and a $s u(2)_{c}$ Wess-ZuminoNovikov-Witten Conformal Field Theory (CFT), in the singular limit when the central charge is infinite; the RS wave functions solve the Knizhnik-Zamolodchikov equations for the CFT correlation functions. The results of Sierra are indeed related to the connection existing between models in statistical mechanics and correlations functions of a suitable CFT established through the OSBA. In fact the solution of the quasi classical OSBA equations is equivalent to solve the Knizhnik-Zamolodchikov equations [24, 25] and in particular the quasi-classical OSBA equations for the vertex models generate the correlators of the $s u(2)$ Wess-Zumino-Novikov-Witten CFT.

The paper is organized as follows. In section II we review the exact solution of the pairing model. In section III the quasi-classical expansion of the OSBA of the disordered six vertex model is identified as the diagonalization of the BCS model. Section IV is devoted to the conclusions. The connection between the diagonalization of the pairing model for infinite pairing coupling constant $g$ and the diagonalization of Gaudin magnet is reviewed in Appendix A. In the Appendix B we summarize the QISM of the inhomogeneous vertex model.

\section{The exact solution of the pairing Hamiltonian}

In this section we review the exact solution 19, 20] of the BCS model (Eq. (11)) and the formulation of its integrability. Due to the form of the pairing interaction in Eq (1), single occupied states are frozen and we can focus on scattering of pairs. The Schrödinger equation for a state of $N$ Cooper pairs

$$
H|N\rangle=\mathcal{E}|N\rangle
$$

has the solution 19, 20]

$$
\begin{aligned}
|N\rangle & =\prod_{\alpha=1}^{N} \sigma^{+}\left(e_{\alpha}, \varepsilon\right)|0\rangle \quad ; \quad \sigma^{+}\left(e_{\alpha}, \varepsilon\right):=\sum_{j=1}^{\Omega} \frac{\sigma_{j}^{\dagger}}{2 \varepsilon_{j}-e_{\alpha}} \\
\mathcal{E} & =\sum_{\alpha=1}^{N} e_{\alpha}
\end{aligned}
$$

The operators $\sigma_{j}^{-}:=c_{j, \downarrow} c_{j, \uparrow}, \sigma_{j}^{+}=\left(\sigma_{j}^{-}\right)^{\dagger}$ and $\sigma_{j}^{z}:=\left(c_{j, \uparrow}^{\dagger} c_{j, \uparrow}+c_{j, \downarrow}^{\dagger} c_{j, \downarrow}-1\right) / 2$ realize $s u(2)$ in the lowest representation. The vacuum state is the highest weight vector of $s u(2):|0\rangle:=|1 / 2,-1 / 2\rangle$. The operators $\left\{\sigma^{ \pm}\left(e_{\alpha}, \varepsilon\right), \sigma^{z}\left(e_{\alpha}, \varepsilon\right)\right\}$ generate (for generic 
$e_{\alpha}$ ) the Gaudin algebra $\mathcal{G}[\operatorname{sl}(2)]$ (see the Appendix A and Eqs. (15) of the next section). The energy $\mathcal{E}$ is given in terms of the spectral parameters $e_{\alpha}$ which satisfy the algebraic equations 19.

$$
\frac{1}{g}+\sum_{\substack{\beta=1 \\ \beta \neq \alpha}}^{N} \frac{2}{e_{\beta}-e_{\alpha}}-\sum_{j=1}^{\Omega} \frac{1}{2 \varepsilon_{j}-e_{\alpha}}=0, \quad \alpha=1, \ldots, N .
$$

The method employed by RS has analogies with the coordinate BA technique. In fact, in the coordinate BA the ansatz functions are plane waves (describing free particles) modified to include the interaction. In the RS solution the ansatz functions are the solutions of the model when pairs of time-reversed electrons are treated as bosons; these functions are modified because Cooper pairs behave as hard-core bosons. In both the RS and the BA procedures the modification enters the set of the algebraic equations for the rapidities (Bethe equations) parameterizing the eigenvalues of the Hamiltonian.

By using the spin realization of pair operators $\left\{\sigma_{j}^{z}, \sigma_{j}^{ \pm}\right\}$, the pairing Hamiltonian can be written as a quantum spin model with long range interaction in a non uniform fictitious magnetic field, given by $\varepsilon_{j}$

$$
H=\sum_{j=1}^{\Omega} 2 \varepsilon_{j} \sigma_{j}^{z}-\frac{g}{2} \sum_{j, l=1}^{\Omega}\left(\sigma_{l}^{+} \sigma_{j}^{-}+\sigma_{j}^{+} \sigma_{l}^{-}\right)+\text {const . }
$$

Cambiaggio et. al. [22 found that the integrals of motion $\tau_{j}$ of this model, if $\varepsilon_{j} \neq \varepsilon_{l}$, $\forall j \neq l$ have the form

$$
\tau_{j}=\frac{1}{g} \sigma_{j}^{z}-\Xi_{j},
$$

and satisfy the commutation relations $\left[H, \tau_{j}\right]=\left[\tau_{j}, \tau_{l}\right]=0, \forall j, l \in\{1, \ldots, \Omega\}$. The operators $\Xi_{j}$ in Eqs. (6) are spin-1/2 Gaudin Hamiltonians [8]

$$
\Xi_{j}:=\sum_{\substack{l=1 \\ l \neq j}}^{\Omega} \frac{\boldsymbol{\sigma}_{j} \cdot \boldsymbol{\sigma}_{l}}{\varepsilon_{j}-\varepsilon_{l}}
$$

The commuting operators $\tau_{j}$ were also found by Sklyanin [11] by taking the quasiclassical limit of the monodromy matrix of the inhomogeneous vertex model twisted by a term proportional to $\sigma_{j}^{z} / g$. The pairing Hamiltonian can be expressed as function of the integrals of motion as

$$
\frac{1}{g^{3}} H=\frac{1}{g^{2}} \sum_{j=1}^{\Omega} 2 \varepsilon_{j} \tau_{j}+\sum_{j, l=1}^{\Omega} \tau_{j} \tau_{l}+\text { const. }
$$

In the limit $g \rightarrow \infty$ the problem is equivalent to the diagonalization of (all) the Gaudin Hamiltonians (see the Appendix A for details).

\section{The OSBA of the inhomogeneous vertex model and the pairing model}

Vertex models are two dimensional classical models which were solved long ago by inverse methods [12. Generalizations to $s u(2)$ higher representations and to include disorder were intensively studied 14, 15, 16].

In this section we introduce a vertex model in which the inhomogeneity is due to the combination of given (see below) disordered distribution of both the spin and the 


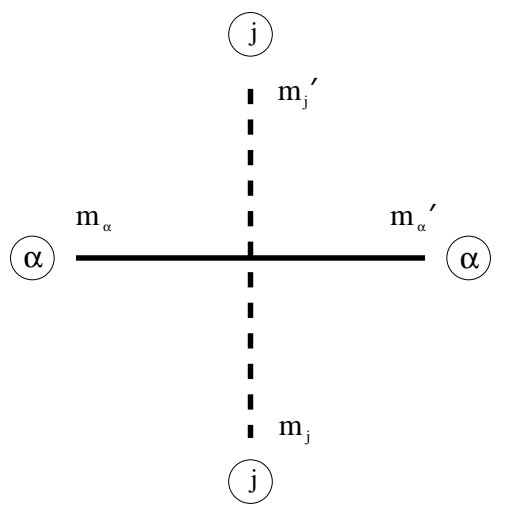

Figure 1. The vertex $(\alpha, j) \in \Lambda$. Disorder $z_{j}$ is distributed along the vertical lines. It is due to both the spin inhomogeneity and impurities $I_{j}$ distributed in the lattice: $z_{j} \equiv z\left(S_{j}\right)+z\left(I_{j}\right)$

impurities in the lattice. Then we apply the scheme developed by Babujian et. al. 17] to relate this inhomogeneous vertex models to the BCS model Eq. (11).

The model is defined in the following way. On the edges of the square lattice $\Lambda: N_{v} \times N_{h}\left(N_{v}\right.$ columns and $N_{h}$ rows $)$ are arranged $N_{v}+1$ types of spin variables. On horizontal edges (labelled by $\alpha=1 \ldots N_{h}$ ) are arranged the spins $\sigma$ taking spin projection $m_{\alpha} \in\{ \pm 1 / 2\}$. On the columns (labelled by $j=1 \ldots N_{v}$ ) the spin variables $S_{j}$ can take any value $m_{j} \in\left\{-s_{j}, \ldots,+s_{j}\right\}$ of the $s_{j}$-th representation of $s u(2)$. The partition function is restricted to configurations for which an even number of spins are into (or out of) each lattice site (vertex); configurations in which the four spins are all in or all out are excluded (ice rule). The "scattering" between spin states $\left(m_{\alpha}, m_{j}\right) \rightarrow\left(m_{\alpha}^{\prime}, m_{j}^{\prime}\right)$ of vertex $(\alpha, j)$ (see Fig. 1) have weights fixed by the universal matrix elements $R_{m_{\alpha}, m_{\alpha}^{\prime}}^{m_{j}, m_{j}^{\prime}}\left(\lambda-z_{j}\right)$ where $\lambda$ is the spectral parameter. The quantities $z_{j}$ (see also Ref. 15]) shift spectral parameters as inhomogeneities which we assume distributed only along the columns of $\Lambda$ (Fig. 1).

The variables $z_{j}$ take into account of disorder induced by the mixture of spin representations and/or by the actual distribution of impurities $I_{j}$ in $\Lambda$ : $z_{j} \equiv$ $z\left(S_{j}\right)+z\left(I_{j}\right)$. We assume that both $z\left(S_{j}\right)$ and $z\left(I_{j}\right)$ enter the universal matrix (see Eq. (B.1) ) in the same functional form. The disordered six vertex model corresponds to the choice: $z\left(S_{j}\right)=0, z\left(I_{j}\right) \neq 0$. We impose periodic boundary conditions.

The transfer matrix $T(\lambda \mid \mathbf{z})$, where $\mathbf{z}:=\left(z_{1} \ldots z_{N_{h}}\right)$, can be expressed in terms of rational $R$-matrices $R_{X}, X=\{\sigma, S\}$ (see Eq. (B.1)) which fulfill Yang Baxter relations (see the Appendix Appendix B). This implies the integrability of the model: $[T(\lambda \mid \mathbf{z}), T(\mu \mid \mathbf{z})]=0$.

The application of the transfer matrix to the Bethe vector $\Phi\left(\lambda_{1} \ldots \lambda_{N} \mid \mathbf{z}\right)$ reads:

$$
\begin{array}{r}
T(\lambda \mid \mathbf{z}) \Phi\left(\lambda_{1} \ldots \lambda_{N} \mid \mathbf{z}\right)=\Lambda\left(\lambda, \lambda_{1} \ldots \lambda_{N} \mid \mathbf{z}\right) \Phi\left(\lambda_{1} \ldots \lambda_{N} \mid \mathbf{z}\right) \\
-\sum_{\alpha=1}^{N} \frac{F_{\alpha}}{\lambda-\lambda_{\alpha}} \Phi_{\alpha}\left(\lambda_{1} . . \lambda_{\alpha-1}, \lambda, \lambda_{\alpha+1} . . \lambda_{N} \mid \mathbf{z}\right),
\end{array}
$$

(for the explicit form of the quantities $T(\lambda \mid \mathbf{z}), \Phi\left(\lambda_{1} \ldots \lambda_{N} \mid \mathbf{z}\right), \Lambda\left(\lambda, \lambda_{1} \ldots \lambda_{N} \mid \mathbf{z}\right)$, $F_{\alpha}$, and $\Phi_{\alpha}\left(\lambda_{1} . . \lambda_{\alpha-1}, \lambda, \lambda_{\alpha+1} . . \lambda_{N} \mid \mathbf{z}\right)$ in (9), see Ref. [17]). The condition for the diagonalization of $T$ (and of the constants of motion generated by $T$ ) is that 
the spectral parameters are chosen to cancel the "unwanted terms" (the second contribution to Eq. (9)) in the spectral problem (9); a sufficient condition is: $F_{\alpha}=0$ (algebraic BA equations). Such a condition has been termed as "Mass-Shell" constraint 17] imposed on Eq. (9). The OSBA spectral problem, instead, arises when the "unwanted terms" are considered in Eq. (9); the spectral parameters obey a new set of equations called OSBA equations (see Eq. (13) below). unfixed). The statement of the OSBA problem has been shown remarkable in the quasi-classical limit. In fact, it was discovered that the solutions of the quasi-classical OSBA problem satisfy the Knizhnik-Zamolodchikov equations [24, 25] for the $s u(2)$ CFT. In the following we shall see how the quasi-classical limit of the OSBA problem for the disordered vertex model is solved by spectral parameters fulfilling the Eqs. (4).

The quasi-classical limit of the vertex model is obtained through the expansion of $R_{X}(x ; \eta)$ in powers of $\eta\left(R_{X}(x ; 0)\right.$ is the identity).

Using the expressions for the monodromy, transfer, and universal matrices Eqs. (B.1), (B.3), the quasi-classical limit of the OSBA Eq. (9) reads

$$
\sum_{j=1}^{N_{v}} \frac{H_{j}}{\lambda-z_{j}} \phi=h \phi+\sum_{\alpha=1}^{N} \frac{f_{\alpha}}{\lambda-\lambda_{\alpha}} \phi_{\alpha},
$$

up to order $\mathcal{O}\left(\eta^{N+2}\right)$, where the explicit form of $h$ and $\phi_{\alpha}$ in $(10)$ is given in the Ref. [17]. By integrating Eq. (10) on a closed loop in the complex $\lambda$-plane encircling the pole $\lambda=z_{j}$ we obtain

$$
\begin{aligned}
& H_{j} \phi=h_{j} \phi+\sum_{\alpha=1}^{N} \frac{f_{\alpha}}{z_{j}-\lambda_{\alpha}} S_{j}^{+} \phi_{\alpha}^{\prime}, \\
& h_{j}=\sum_{\substack{l=1 \\
l \neq j}}^{N_{v}} \frac{s_{l} s_{j}}{z_{l}-z_{j}}-\sum_{\alpha=1}^{N} \frac{s_{j}}{\lambda_{\alpha}-z_{j}}, \quad j=1 \ldots N_{v}, \\
& f_{\alpha}=\sum_{\substack{\beta=1 \\
\alpha \neq \beta}}^{N} \frac{1}{\lambda_{\alpha}-\lambda_{\beta}}-\sum_{j=1}^{N_{v}} \frac{s_{j}}{\lambda_{\alpha}-z_{j}}, \quad \alpha=1 \ldots N .
\end{aligned}
$$

The Bethe vectors in the quasi-classical limit are

$$
\phi:=\prod_{\beta=1}^{N} S^{+}\left(\lambda_{\beta}, z\right)|v a c\rangle, \quad \phi_{\alpha}^{\prime}:=\prod_{\substack{\beta=1 \\ \beta \neq \alpha}}^{N} S^{+}\left(\lambda_{\beta}, z\right)|v a c\rangle,
$$

Here $|v a c\rangle=\otimes_{j=1}^{N_{v}}\left|s_{j},-s_{j}\right\rangle$, where $S_{j}^{-}\left|s_{j},-s_{j}\right\rangle=0$, i.e. $|v a c\rangle$ is the highest weight vector in $\otimes_{j} s u(2)_{j}$. The three operators $S^{ \pm, z}\left(\lambda_{\beta}, z\right):=\sum_{j=1}^{N_{v}} S_{j}^{ \pm, z} /\left(z_{j}-\lambda_{\beta}\right)$ generate higher dimensional representations of the Gaudin algebra $\mathcal{G}[s l(2)]$, given by (see also Eq. (A.2))

$$
\begin{aligned}
{\left[S^{z}\left(\lambda_{\alpha}, z\right), S^{ \pm}\left(\lambda_{\beta}, z\right)\right] } & = \pm \frac{S^{ \pm}\left(\lambda_{\alpha}, z\right)-S^{ \pm}\left(\lambda_{\beta}, z\right)}{\lambda_{\beta}-\lambda_{\alpha}} \\
{\left[S^{+}\left(\lambda_{\alpha}, z\right), S^{-}\left(\lambda_{\beta}, z\right)\right] } & =\frac{S^{z}\left(\lambda_{\alpha}, z\right)-S^{z}\left(\lambda_{\beta}, z\right)}{\lambda_{\beta}-\lambda_{\alpha}}
\end{aligned} .
$$

The "Mass-Shell" constraint: $f_{\alpha}=0$ corresponds to the diagonalization of the Gaudin model (see Appendix A). 
The solution of the spectral problem for the pairing model is recovered substituting

$$
f_{\alpha}=\frac{1}{2}\left(\sum_{j=1}^{N_{v}} \frac{1-2 s_{j}}{\lambda_{\alpha}-z_{j}}+\frac{1}{g}\right), \quad \alpha=1 \ldots N,
$$

in the left hand side of Eqs. (13). In fact, the resulting equations coincide with Eqs. (14). Substituting Eqs. (15) in (11), and summing over index $j=1 \ldots N_{v}$ we obtain

$$
\sum_{\alpha=1}^{N}\left(\sum_{j=1}^{N_{v}} \frac{4 s_{j}-1}{\lambda_{\alpha}-z_{j}}+\frac{1}{g}\right) \phi=0,
$$

where we have used the fact that: $\sum_{j=1}^{N_{v}} h_{j}=-\sum_{\alpha=1}^{N} \sum_{j=1}^{N_{v}} s_{j} /\left(\lambda_{\alpha}-z_{j}\right)$. Eq. (16) shows that, the OSBA spectral problem is transformed in a spectral problem involving only diagonal matrix elements of suitably shifted (by $f_{\alpha}$ ) transfer matrix of the vertex model (in the quasi classical limit).

Since the limit $g \rightarrow \infty$ should correspond to the same result of $f_{\alpha} \rightarrow 0$ for generic $s_{j}$ (compare with Eqs (A.4)), we impose that the distribution of spins $S_{j}$ through the lattice fulfills the condition

$$
\sum_{j=1}^{N_{v}} \frac{\left(1-2 s_{j}\right)}{\left(\lambda_{\alpha}-z_{j}\right)} \equiv 0 .
$$

In this case Eq. (15) reduces to

$$
f_{\alpha}=\frac{1}{2 g}, \quad \alpha=1 \ldots N \text {. }
$$

We choose $s_{j}=1 / 2 \quad \forall j$ in order to fulfill Eq. (17): the inhomogeneous vertex model becomes the disordered six vertex model since $z\left(S_{j}\right)=0$ and $z\left(I_{j}\right) \neq 0$. This implies that

$$
H_{j} \equiv \Xi_{j}, \text { and } \phi \equiv|N\rangle,
$$

where: $N_{v}=\Omega$ (compare with Eqs (3), (可). Eq. (16) can be transformed in the following eigenvalue equation:

$$
\begin{aligned}
& \sum_{j=1}^{\Omega} \sum_{\alpha=1}^{N}\left(\frac{-\sigma_{j}^{z}}{2 \varepsilon_{j}-e_{\alpha}}-\frac{1}{N} \Xi_{j}\right) \phi=\sum_{j=1}^{\Omega} \sum_{\alpha=1}^{N} \tau_{j, \alpha} \phi, \\
& \tau_{j, \alpha}:=\left(\frac{1}{\Omega g}-\frac{1}{N} h_{j}\right),
\end{aligned}
$$

where Eqs. (1) (or (13), (18) ), (12), and: $\sum_{\beta=1}^{N} \sigma^{z}\left(e_{\alpha}, \varepsilon\right) \phi=1 / 2 \sum_{\beta=1}^{N} \sum_{j=1}^{\Omega} 1 /\left(e_{\alpha}-\right.$ $\left.2 \varepsilon_{j}\right) \phi$ have been used (the parameters in Eqs. (12)-20) are redefined as $z_{j} \leftrightarrow$ $2 \varepsilon_{j}, \lambda_{\alpha} \leftrightarrow e_{\alpha}$ ). We point out that quantities in (20) are the eigenvalues of operators $\tau_{j}$ in Eq. (6) for generic $\Omega / N$. At "half filling" $\Omega=2 N$ Eq. (20) reduces to

$$
\tau_{j, \alpha}=\frac{1}{\Omega}\left(\frac{1}{g}-2 h_{j}\right),(j=1, \ldots, \Omega) .
$$

Equations (20), (21) coincide with those ones found by Sklyanin [11] and by Sierra 23]. The main result obtained in this paper is the connection between Eqs. (11) and Eqs. (19), (20) through the Eq. (15). The OSBA problem for the disordered six vertex model (which does not account for diagonalizing the transfer matrix of the vertex 
model) reveals the existence of a class of spectral problems (parameterized by $f_{\alpha}$ ) which turns out to be diagonal on the quasi-classical Bethe vectors basis. For $f_{\alpha}$ fixed by Eqs. (18) the diagonalization of the BCS model is obtained.

Furthermore, what we have discussed so far implies that the pairing Hamiltonian can be obtained from functionals of $\tau_{j}$ whose quasi-classical expansions have the following form:

$$
\mathcal{T}(e \mid \mathbf{z})=\sum_{a=0}^{\infty} \eta^{2 a} e^{a-1}\left[\frac{1}{2 g^{2}}+\tau(e)\right]^{a},
$$

with

$$
\tau(e):=\sum_{j=1}^{\Omega} \frac{\tau_{j}}{e-2 \varepsilon_{j}} .
$$

We point out that $\left[\mathcal{T}(e \mid \mathbf{z}), \mathcal{T}\left(e^{\prime} \mid \mathbf{z}\right)\right]=0, \forall e, e^{\prime}$ since quantities $\tau_{j}$ 's commute each other. The residue in the poles $e=2 \varepsilon_{j}$ of the $\eta^{2}$ coefficient provides the integrals of motion $\tau_{j}$. The residue of the $\eta^{4}$ coefficient reads (see Eq. (8))

$$
\sum_{j, l=1}^{\Omega} \tau_{j} \tau_{l}+\frac{1}{g^{2}} \sum_{j=1}^{\Omega} 2 \varepsilon_{j} \tau_{j}=\frac{1}{g^{3}} H
$$

\section{Conclusions}

We have established a novel connection between the disordered six vertex model and the BCS model for generic $g$ through the OSBA procedure. The BCS model is diagonalized by the quasi classical limit of the OSBA equations of the disordered six vertex model. Retaining certain off diagonal terms of the transfer matrix of the vertex model corresponds to the diagonalization of the integrals of motion of the pairing model for finite $g$. The "mass shell" condition (and then the diagonalization of the quasi classical transfer matrix of the vertex model) reproduces the limit $g \rightarrow \infty$; the corresponding problem is the Gaudin spectral problem.

The integrals of motion of the BCS model coincide with the integrals found by Sklyanin 11 by considering a twist in the monodromy matrix of the vertex model (see Eq. B.3). The algebraic equations which diagonalize these integrals of motion via algebraic BA (namely via the Mass Shell BA procedure) coincide with the Richardson's equations. The present study shows that the Sklyanin's procedure produces the same results of the Off Shell BA procedure applied to the untwisted monodromy matrix.

The existence of the relation between BCS model and quasi-classical vertex models, found in the present paper, is consistent with the correspondence between CFT and the BCS model recently found by Sierra 23].

Eqs. (4) were already conjectured by Gaudin (see formulas (5.15), (5.16) of Ref. [8]) as connected (through Jacobian of certain matrices) with the norms of the Bethe vectors, $\operatorname{det}\left(\partial \mathrm{f}_{\alpha} / \partial \mathrm{e}_{\beta}\right) \sim\|\phi\|$ (see Refs. [21], [26]). In this work we have shown that the Jacobian is connected with OSBA of the vertex model. This might be useful to compute norms (and scalar products) and, then, to express the correlation functions of the BCS model as suitable determinants. This exact calculation is our major task in the future. 
The BCS model and the off shell Bethe ansatz for vertex models

\section{Acknowledgments}

We thank G. Sierra for constant and invaluable help since the early stages of this work. A. Osterloh is acknowledged for very useful discussions and for a critical reading of the manuscript. We thank F. Dolcini and G. Giaquinta for discussions. We acknowledge the financial support of INFM-PRA-SSQI and the European Community (Contract FMRX-CT-97-0143).

\section{Appendix A. The pairing model and the Gaudin spectral problem}

In this Appendix we discuss the connection between the pairing model and the Gaudin model.

The limit $g \rightarrow \infty$ of the constants of motion $\tau_{j}$ (河) coincides with Hamiltonians $\Xi_{j}$. Since Eq. (6), the spectrum of the pairing problem coincide with that one of the Gaudin magnet [8]: $\Xi(u):=\sum_{j=1}^{\Omega} \Xi_{j} /\left(u-2 \varepsilon_{j}\right)$ (u is a complex parameter). The total energy is: $h(u):=\sum_{j=1}^{\Omega} h_{j} /\left(u-2 \varepsilon_{j}\right)\left(h_{j}\right.$ is fixed by Eq. (12)). The Bethe vectors of the Gaudin and the pairing problems coincide formally for any $g$ since operators $\left(\sigma^{ \pm}(u, \varepsilon), \sigma^{z}(u, \varepsilon)\right)$ in (3) generate the Gaudin algebra $\mathcal{G}[s l(2)]$ in the lowest representation:

$$
\begin{aligned}
{\left[\sigma^{z}(u, \varepsilon), \sigma^{ \pm}(w, \varepsilon)\right] } & = \pm \frac{\sigma^{ \pm}(u, \varepsilon)-\sigma^{ \pm}(w, \varepsilon)}{w-u}, \\
{\left[\sigma^{+}(u, \varepsilon), \sigma^{-}(w, \varepsilon)\right] } & =\frac{\sigma^{z}(u, \varepsilon)-\sigma^{z}(w, \varepsilon)}{w-u},
\end{aligned}
$$

where $\sigma^{z}(u, \varepsilon):=\sum_{j=1}^{\Omega} \sigma_{j}^{z} /\left(2 \varepsilon_{j}-u\right)$.

However, the spectral parameters entering the eigenvectors of the two models satisfy, for generic $g$, different equations (compare with Eqs. (4))

$$
\sum_{\substack{\beta=1 \\ \beta \neq \alpha}}^{N} \frac{2}{e_{\beta}-e_{\alpha}}-\sum_{j=1}^{\Omega} \frac{1}{2 \varepsilon_{j}-e_{\alpha}}=0 .
$$

We point out that Eqs. (A.2) are the limit $g \rightarrow \infty$ of Eqs. (4) for the pairing model. In this limit the two models have the same eigenvectors (see Eqs (3), (4), (A.2)).

Thus the diagonalization of the Gaudin model is equivalent to the diagonalization of the BCS model for $g \rightarrow \infty$.

The limit of large $g$ in Eq. (8) gives:

$$
\begin{aligned}
H \approx & -g \sum_{j, l=1}^{\Omega} \frac{1}{\varepsilon_{j}-\varepsilon_{l}}\left[\left(\varepsilon_{j}+\varepsilon_{l}\right) \sigma_{j}^{z} \sigma_{l}^{z}+\varepsilon_{j} \sigma_{j}^{+} \sigma_{l}^{-}\right] \\
& \equiv-\frac{g}{2} \sum_{j, l=1}^{\Omega} \sigma_{j}^{+} \sigma_{l}^{-},
\end{aligned}
$$

which (consistently) reproduce the Hamiltonian (1) for large $g$.

The QISM was applied to Gaudin magnet for generic spin $\mathbf{S}_{j}$ in Ref. [8, 27]. In this case the Gaudin Hamiltonians are $H_{l}:=\sum_{\substack{j=1 \\ j \neq l}}^{\Omega} \mathbf{S}_{l} \cdot \mathbf{S}_{j} /\left(\varepsilon_{j}-\varepsilon_{l}\right)$. The spectral parameters obey

$$
\sum_{\substack{\beta=1 \\ \beta \neq \alpha}}^{N} \frac{1}{e_{\beta}-e_{\alpha}}-\sum_{j=1}^{\Omega} \frac{s_{j}}{2 \varepsilon_{j}-e_{\alpha}}=0,
$$


where $-s_{j}$ corresponds to the highest weight vector of $\mathbf{S}_{j}$.

\section{Appendix B. Integrability of the inhomogeneous vertex model}

In this appendix we discuss the QISM of the inhomogeneous vertex model together with its quasi-classical expansion.

The universal matrix of the model reads

$$
R_{X}(\lambda-z ; \eta)=\mathbb{1} \otimes \mathbb{1}+f(\lambda-z, \eta) \sigma \otimes X,
$$

where $f(x, \eta):=2 \eta /(\eta-2 x)$ depending on the arbitrary parameter $\eta \in \mathbb{R}$. The physical $R$-matrix of the model corresponds to take $X \equiv S$ in (B.1); the auxiliary one corresponds to $X \equiv \sigma$ and $z=0$. Both these matrices fulfill homogeneous $(S \equiv \sigma)$ Yang-Baxter relations; in addition they fulfill the the inhomogeneous $(S \neq \sigma)$ one 14]:

$$
\begin{aligned}
R_{\sigma}^{12}(\lambda-\mu) R_{S}^{23}(\lambda-z) & R_{S}^{12}(\mu-z)= \\
& R_{S}^{23}(\mu-z) R_{S}^{12}(\lambda-z) R_{\sigma}^{23}(\lambda-\mu),
\end{aligned}
$$

where $R^{12} \doteq R \otimes \mathbb{1}$ and $R^{23} \doteq \mathbb{1} \otimes R$ act on the vector space $V_{1} \otimes V_{2} \otimes V_{3} ; R^{12}$ and $R^{23}$ act as the identity in the vector spaces 3 and 1 respectively. Eq. (B.2) is the sufficient condition for which the model, can be solved by diagonalizing the column to column (which is $2 \times 2$ matrix in operators $S_{j}$ ) transfer matrix (instead of the row to row one which is $\left.\left(2 s_{j}+1\right) \times\left(2 s_{j}+1\right)\right)$ obtained through the trace in the two dimensional horizontal vector space (which is spanned by spins along the rows of $\Lambda$ ) labelled by "(0)":

$$
T(\lambda \mid \mathbf{z}):=\operatorname{tr}_{(0)} J(\lambda \mid \mathbf{z}) .
$$

Twisted monodromy matrix is obtained by $J(\lambda \mid \mathbf{z}) \rightarrow e^{a \sigma_{j}^{z}} J(\lambda \mid \mathbf{z})$. The transfer matrices commute at different values of spectral parameters: $[T(\lambda \mid \mathbf{z}), T(\mu \mid \mathbf{z})]=0$ (it proves the integrability of the model) since the monodromy matrix $J(\lambda \mid \mathbf{z}):=$ $\prod_{j=N_{v}}^{1} R_{S_{j}}^{(0)}\left(\lambda-z_{j}\right)$ satisfies: $R_{\sigma}(\lambda-\mu)[J(\lambda \mid \mathbf{z}) \otimes J(\mu \mid \mathbf{z})]=[J(\mu \mid \mathbf{z}) \otimes J(\lambda \mid \mathbf{z})] R_{\sigma}(\lambda-\mu)$ (induced by Eq. (B.2). The matrices $R_{S_{j}}^{(0)}$ and $R_{\sigma}$ are

$$
\begin{aligned}
R_{S_{j}}^{(0)}(x, \eta):= & \left(\begin{array}{cc}
\mathbb{1}+f(x, \eta) S_{j}^{z} & f(x, \eta) S_{j}^{-} \\
f(x, \eta) S_{j}^{+} & \mathbb{1}-f(x, \eta) S_{j}^{z}
\end{array}\right), \\
R_{\sigma}(\lambda-\mu, \eta):= & \left(\begin{array}{cccc}
1 & 0 & 0 & 0 \\
0 & c & b & 0 \\
0 & b & c & 0 \\
0 & 0 & 0 & 1
\end{array}\right)
\end{aligned}
$$

where: $b(\lambda-\mu):=\eta /(\eta-\lambda-\mu), c(\lambda-\mu):=\lambda-\mu /(\lambda-\mu-\eta)$ (note that $R_{\sigma}(\lambda-\mu, \eta$ ) is $z$-independent).

In the quasi-classical limit, the system generates a hierarchy of integrable systems in the quasi-classical limit since

$$
\sum_{a=b+c=0}^{\infty}\left[T_{b}(\lambda \mid \mathbf{z}), T_{c}(\mu \mid \mathbf{z})\right]=0
$$


where we have used the $\eta$-expansion of the transfer matrix: $T(\lambda \mid \mathbf{z})=\sum_{a=0}^{\infty} \eta^{a} T_{a}(\lambda \mid \mathbf{z})$ (the sum in Eq. (B.6) is meant on ordered partitions of $a$ including $b \vee c=0$ ). Up to order $\eta^{2}$, the transfer matrix reads

$$
T(\lambda \mid \mathbf{z})=2 \mathbb{1}+2 \eta^{2} \sum_{j=1}^{N_{v}} \frac{H_{j}}{\lambda-z_{j}},
$$

where the Hamiltonians $H_{j}$ in Eq. (B.7) are

$$
H_{j}=\sum_{\substack{l=1 \\ l \neq j}}^{N_{v}} \frac{\mathbf{S}_{l} \cdot \mathbf{S}_{j}}{z_{j}-z_{l}}, \quad\left(j=1, \ldots, N_{v}\right) .
$$

The transfer matrix (B.7) coincide with the quasi-classical expansion of the twisted Gaudin model's transfer matrix (see formula (1.16) of the Ref. [11).

\section{References}

[1] J. Bardeen, L.N. Cooper, and J.R. Schrieffer, Phys. Rev. 108, 1175 (1957)

[2] M. Tinkham, Introduction to Superconductivity, (McGraw-Hill, New York, 1996) (second edition).

[3] F. Iachello, Nucl. Phys. A 570, 145c (1994).

[4] D.H.Rischke and R. D. Pisarski, proceedings of the "Fifth Workshop on QCD", Villefranche, nucl-th/0004016.

[5] C.T. Black, D.C. Ralph, and M. Tinkham, Phys. Rev. Lett. 74, 32 (1995); ibidem 76, 688 (1996); ibidem 78, 4087 (1997).

[6] K.A. Matveev and A.I. Larkin, Phys. Rev. Lett. 78, 3749 (1997); A. Mastellone, G. Falci, and R. Fazio, Phys. Rev. Lett. 80, 4542 (1998); J. von Delft and D.C. Ralph, to be published in Physics Reports.

[7] H. Kleinart, Fort. der Physik 26 565, (1978)

[8] M. Gaudin, J. Phys. 37, 1087 (1976)

[9] V.E. Korepin, N.M. Bogoliubov, and A.G. Itzergin, Quantum Inverse Scattering Method and Correlation Functions, (Cambridge University Press, Cambridge, 1993)

[10] L.D. Faddeev and L.A. Takhtajan, Hamiltonian methods in the theory of solitons (Springer, Berlin, 1987)

[11] The same set of operators already emerged from the quasiclassical expansion of the twisted monodromy matrix of the inhomogeneous vertex model; E.K. Sklyanin, J. Sov. Math. 47, 2473 (1989)

[12] R. Baxter, Exactly Solved Models in Statistical Mechanics, (Academic Press, London, 1982)

[13] The inhomogeneous vertex models are related to the Gaudin models [11, 17]: the BA equations of the Gaudin model can be obtained by taking the quasi-classical limit of the BA equations of vertex models.

[14] H.M. Babujian, Nucl. Phys. B 215, 317 (1983)

[15] H.J. de Vega, Nucl. Phys. B 240, 495 (1984)

[16] P.P. Kulish and N. Reshetikhin, J. Phys. A 16, L591 (1983)

[17] H.M. Babujian, J. Phys. A 26, 6981 (1993); H.M. Babujian and R. Flume, hep-th/9310110

[18] The OSBA deals with the off diagonal terms generated by the application of the transfer matrix to the Bethe vectors [17].

[19] R.W. Richardson and N. Sherman, Nucl. Phys. 52, 221 (1964); 52, 253 (1964)

[20] M. Gaudin, Travaux de Michel Gaudin. Modèles exactement résolus, (Les Èditions de Physique, 1995)

[21] R.W. Richardson, J. Math. Phys. 6, 1034 (1965)

[22] M.C. Cambiaggio, A.M.F. Rivas, and M. Saraceno, Nucl. Phys. A 624, 157 (1997)

[23] G. Sierra, Nucl. Phys. B 572, 517 (2000)

[24] B. Feigin, E. Frenkel, and N. Reshetikhin, Comm. Math. Phys. 166, 27 (1994)

[25] V.G. Knizhnik and A.B. Zamolodchikov, Nucl. Phys. B 247, 83 (1984)

[26] V.E. Korepin, Comm. Math. Phys. 86, 391 (1982)

[27] M. Gaudin, La fontion d'onde de Bethe (Masson, Paris, 1983) 